\title{
Gravitational Wave and Transparent Crystal Black Hole
}

\author{
Zhiliang Cao ${ }^{1,2 *}$, Henry Gu Cao ${ }^{3}$ \\ ${ }^{1}$ Wayne State University, Detroit, MI, USA \\ ${ }^{2}$ Shanghai Jiao Tong University, Shanghai, China \\ ${ }^{3}$ Northwestern University, Evanston, IL, USA \\ Email: *williamcao12252000@yahoo.com
}

How to cite this paper: Cao, Z.L. and Cao, H.G. (2016) Gravitational Wave and Transparent Crystal Black Hole. Open Access Library Journal, 3: e3038.

http://dx.doi.org/10.4236/oalib.1103038

Received: September 5, 2016

Accepted: October 15, 2016

Published: October 19, 2016

Copyright $\odot 2016$ by authors and Open Access Library Inc.

This work is licensed under the Creative

Commons Attribution International

License (CC BY 4.0).

http://creativecommons.org/licenses/by/4.0/

\section{(c) (†) Open Access}

\begin{abstract}
This paper uses the results of the latest Unified Field Theory (UFT) and the experimental results of the recent experiments of gravitational wave to study the configuration of a black hole. We conclude that a black hole has no singularity state. Instead, particles in a black hole form a stable system. Topologically, octahedron particles form sphere with two poles. A black hole always releases energy at the poles. If a black hole has an accretion disk, the particles from disk will wrap around the black hole, twist upwards, accelerated by out-going black hole energy at the poles, and ejected out at the top of a pole to form a black hole jet. The predictions made in the paper provide valuable insight regarding the inner workings of the black hole. We hope that our work can help future studies regarding gravitational waves, black hole, and dark energy.
\end{abstract}

\section{Subject Areas}

Particle Physics, Theoretical Physics

Keywords

Dark Matter, Astronomy, Black Hole, Gravitational Wave

\section{Introduction}

Unified Field Theory (UFT) [1]-[11] considers that gravity is the result of space-time distortion by energy. It supports the gravity equation as follow:

$$
\begin{aligned}
& G M=R V^{2} \\
& R=G M / V^{2} .
\end{aligned}
$$


In recent test results [12]-[16], the final mass of the combined mass of merged black hole is 62 solar masses. If $V=C$, the frequency calculated based on the above equations will be: $521 \mathrm{~Hz}$.

For the twin black holes, the frequency is doubled: $1042 \mathrm{~Hz}$.

The actual test result [16] (Figure 1) is that the max frequency is $336 \mathrm{~Hz}$.

Assume that the rotation speed is:

$$
V=(1 / 2)^{1 / 2} C
$$

and:

$$
G M=(1 / 2) R C^{2}
$$

The frequency is changed to:

$$
(1 / 2)^{3 / 2} \times 1025.4=362.5 \mathrm{~Hz} .
$$

According to the test result, the twin black hole failed to maintain the orbit. In 4 cycles, they collided.

$$
362.5 /(1+1 /(4 \times 3.14))=336
$$

When two black holes merge, the circulation speed is:

$$
V=(1 / 2)^{1 / 2} C \text {. }
$$

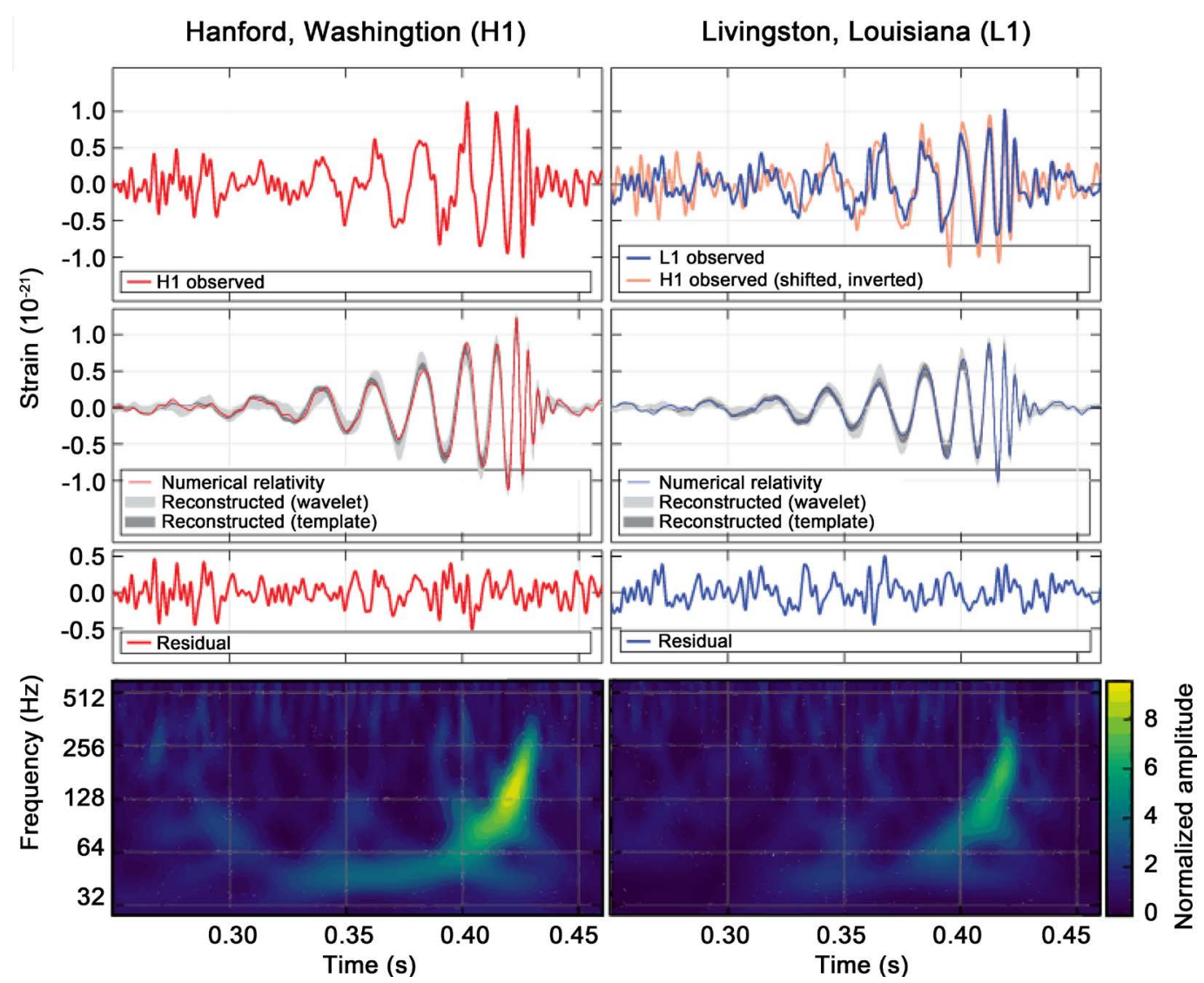

Figure 1. Gravitational wave experiment results. 
When particles circling around the black hole:

$$
G M=R V^{2}
$$

or:

$$
G M=(1 / 2) R C^{2}
$$

\section{Results}

\subsection{Black Hole Internal Structure}

The structural formula [12] for proton is (in unit of electron mass):

$$
2(2 \times 3 \times 5)^{2}+2 \times 3 \times 5+2 \times 3
$$

In order to resist gravitational pressure in a black hole shell, the particle has to be stable. When the mass of the particle in unit of electron mass is divisible by strong interaction energy 137 instead of $(2 \times 3 \times 5)^{2}$, the particle is perfectly in resonance with strong force and most stable (in unit of electron mass). The known particles do not have such characteristics. Since the know particles have three axes in Figure 2, the formula will start with:

$$
3 \times X
$$

A stable strong interacted axis is neutral. Since component with mass of 137 is charged. To make it both divisible and neutral, $X$ can be:

$$
\begin{aligned}
& 2 n \times 137^{(2 m-1)} \text { or } n \times 137^{(2 m)} \\
& n, m=1,2,3, \ldots \\
& 3 \times X: \\
& 3 \times\left(2 n \times 137^{(2 \mathrm{~m}-1)}\right) \text { or } 3 \times\left(n \times 137^{(2 m)}\right) \\
& \text { i.e.: } \\
& \ldots \\
& 3 \times\left(4 \times 137^{2}\right) \\
& 3 \times\left(3 \times 137^{2}\right) \\
& 3 \times\left(2 \times 137^{2}\right) \\
& 3 \times\left(137^{2}\right) \\
& 3 \times(128 \times 137) \\
& 3 \times(64 \times 137) \\
& 3 \times(32 \times 137) \\
& 3 \times(16 \times 137) \\
& 3 \times(8 \times 137) \\
& 3 \times(4 \times 137) \\
& 3 \times(2 \times 137)
\end{aligned}
$$

\subsection{Dark Matter}

Any structure in following formula (in unit of electron mass):

$$
3 \times\left(2 n \times 137^{(2 m-1)}\right) \text { or } 3 \times\left(n \times 137^{(2 m)}\right) .
$$


Can silently interact with any structure in above formula without releasing energy:

$$
3 \times(2 \times 137)+3 \times(2 \times 137) \rightarrow 3 \times(4 \times 137) .
$$

Three different particles:

$$
2 \times(3 \times(128 \times 137)+3 \times(8 \times 137))+3 \times(2 \times 137) \rightarrow 3 \times\left(2 \times 137^{2}\right) .
$$

The interaction without releasing energy leads to the "dark" character of the above structures.

When the neighboring particles share the same structure, they are in resonance. The neighbor particles in a shell should have the same structure.

The particles do not bind together with strong force. They form perfect crystal layers with no friction between layers. In Figure 3, the crystal planes are parallel to the surface of the black hole.

\subsection{Particle Orientation and Sphere}

When a particle moves down toward the core of a black hole, the gravity accelerates the particle. It will collide with a particle and stops. If one of the axis of the octahedron dark particle is parallel to the axis of the black hole, the collision does not cause particles to spin. Therefore, one of the axis of the octahedron dark particle is parallel to the axis of the black hole.

Two neighboring particles vertical to the black hole axis are colliding due to the heat. For the same reason, their axes are lined up, one axis along Longitude, and one axis along Latitude.

We predict that the movements vertical to the axis are heat movements. A simple movement can be in form of vibration along axes. Such heat vibration vertical to the axis create spheres. The heat vibration generates pressure in the spheres and cancels out gravity introduced pressure within a sphere.

A stable black hole has no pressure between shells in the stable areas.

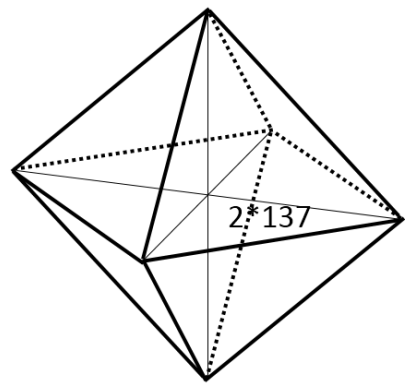

Figure 2. Particles in black hole.
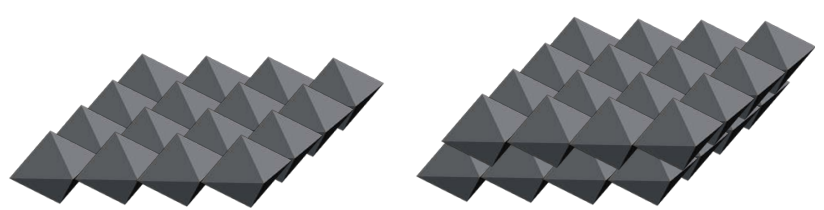

Figure 3. Black hole crystal. 


\subsection{Particle Speed in the Sphere and Heat}

The internal collision of the sphere at the equator should cancel out the gravity of half sphere.

Assume that:

$D$ : density.

$r_{p}: 0.84 \times 10^{-16} \mathrm{~m}$, radius of particle.

$r$ radius of sphere.

Gravity:

$$
F=\int_{0}^{\pi / 2} G 2 \pi r^{2} D \cos \theta d \theta / r^{2}=2 \pi G D
$$

Collision:

$$
F=\left(2 \pi r \times r_{p} D \times\left(V^{2} / 2\right)\right) / r_{p}=2 \pi r \times D \times\left(V^{2} / 2\right)
$$

or:

$$
\begin{aligned}
& 2 \pi r \times D \times\left(V^{2} / 2\right)=2 \pi G D \\
& 2 \pi D G r_{p}=2 \pi r \times r_{p} D \times\left(V^{2} / 2\right) \\
& V^{2}=2 G / r=\left(2 \times 6.67408 \times 10^{-11}\right) / r .
\end{aligned}
$$

If the Sun becomes a black hole, it will be the smallest black hole ever found. The radius:

$$
\begin{aligned}
& r=2950 \mathrm{~m} \\
& V=2.13 \times 10^{-7} \mathrm{~m} / \mathrm{s} .
\end{aligned}
$$

The smallest radius is the radius of proton, or:

$$
r=0.84 \times 10^{-16} \mathrm{~m} \text {. }
$$

$V$ reaches to the maximum speed:

$$
V_{\max }=1260 \mathrm{~m} / \mathrm{s} .
$$

Black holes are very cold indeed.

\section{Discussions}

\subsection{Unreachable Event Horizon}

The spheres in the black hole create a stable system. Such system stops further crashing from happening and there will be no path to reach the event horizon. Instead, the escape energy is one half of the inertia energy, a stable black hole follows:

$$
G M=(1 / 2) R C^{2} \text {. }
$$

\subsection{Transparent Crystal}

The structures:

$$
3 \times(2 n \times 137) \text { or } 3 \times\left(n \times 137^{(m+2)}\right)
$$

are octahedron shaped. 
Topologically, octahedron particle formed sphere Figure 4 has two poles. An octahedron on the sphere has one axis vertical to the sphere, one axis along Longitude, and one axis along Latitude.

The Longitude effects are less in the areas close to two poles. The particles are mainly aligned with the axis of the black hole and the other two axes are aligned by external forces and form a perfect crystal.

The areas further away from the poles have disproportional crystal dislocations that lead to pressures between the shells and form unstable fault structures shaped as squared cylinders/cones. The pressure causes movements along the fault surface. The energy will be released continuously from fault surface as particles crash down toward the core of black hole along the fault surface.

For a single black hole [17] Figure 5, it has both cross shaped bright arms and circular features.

In black hole system [18] in Figure 6, the center pole areas may have ideal crystal surrounded by other cones which are directly map to the bright spots in the real image in Figure 6 on the right.

Due to the gravity, photons release from the deep fault surface are red shifted, while photons from the upper level of black hole are blue (less red shifted).
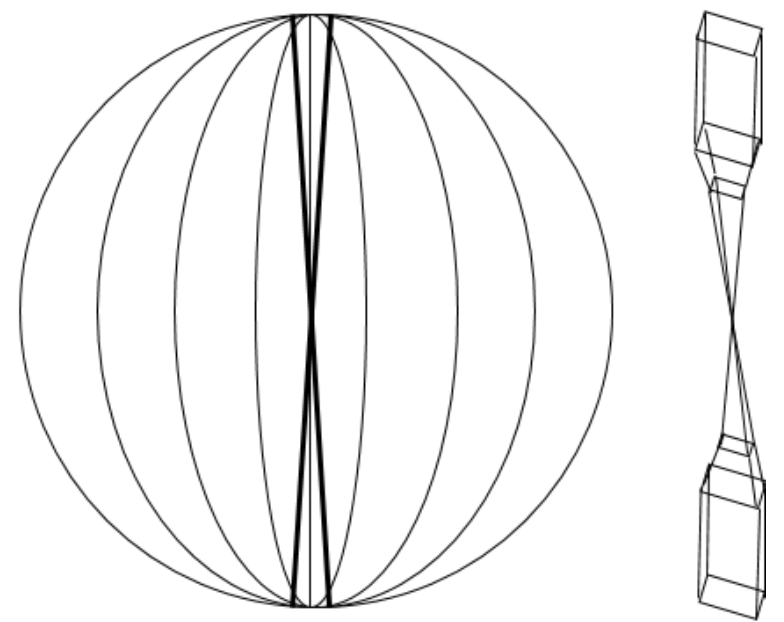

Figure 4. Octahedron particle sphere and fault cone.

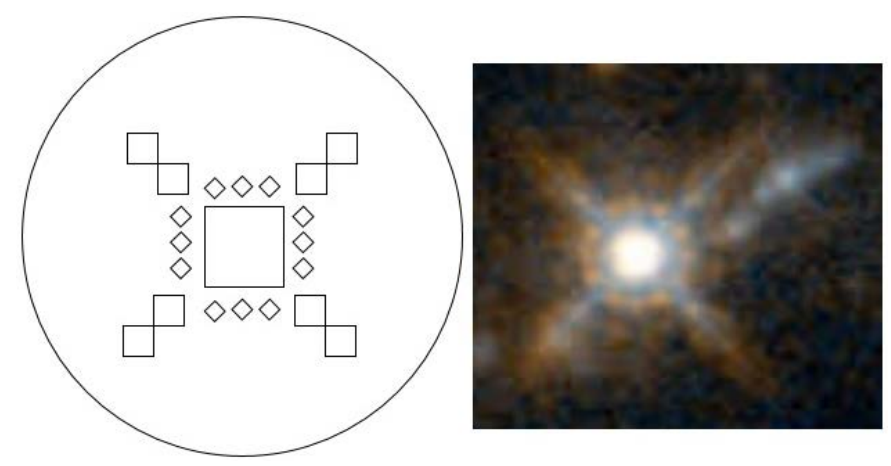

Figure 5. Quasar 1 and defect cones near a pole. 


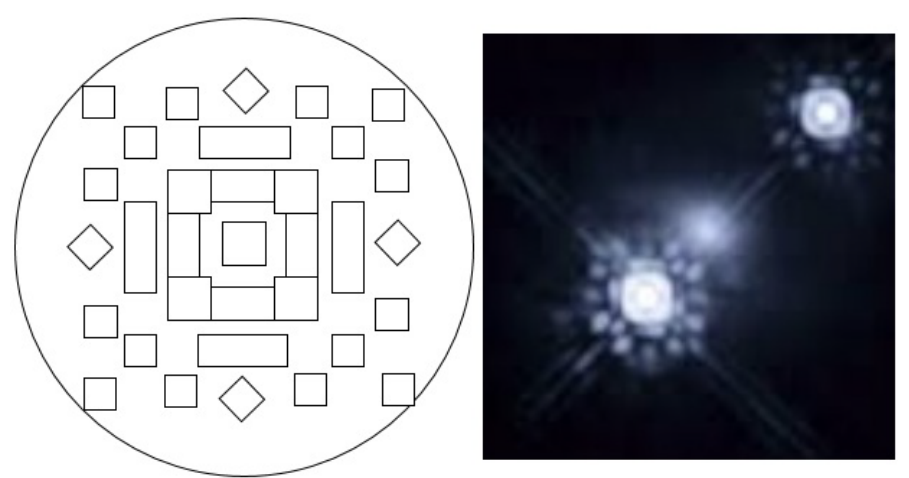

Figure 6. Squared quasar and defect cones near a pole.

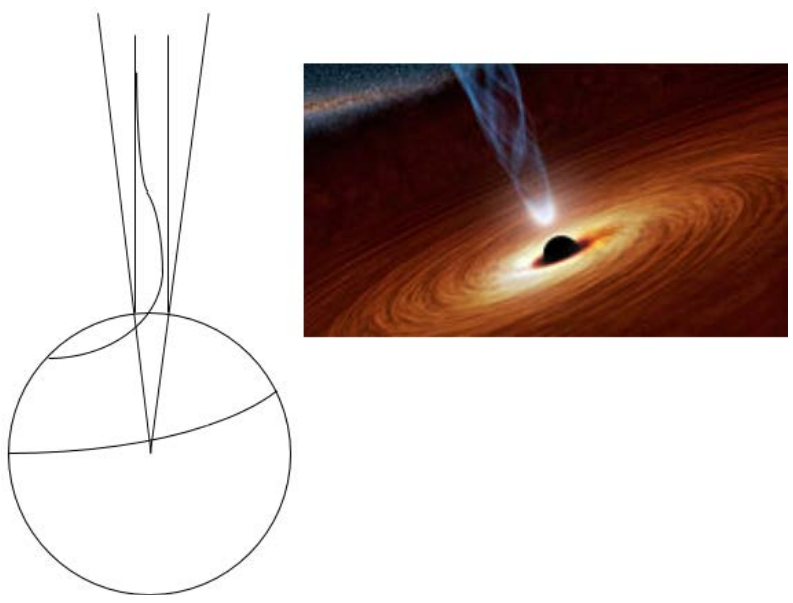

Figure 7. Black hole jet.

\subsection{Black Hole Jet}

When there is an accretion disk, circling matter will touch the surface of black hole, where the vibration movements along a longitude convert the circling matter into layers of spheres wrapping around the black hole. The newly formed spheres twist upwards. When these particles reach a pole, they are accelerated by out-going black hole energy at the poles. The particles are continuously pulled into the new sphere from the rotating disk and ejected at the top of pole. This dynamic process forms a black hole jet Figure 7.

\section{Conclusions}

We predict that:

1) A black hole is composed of dark particles structured as follows:

$$
3 \times\left(2 n \times 137^{(2 m-1)}\right) \text { or } 3 \times\left(n \times 137^{(2 m)}\right), m, n=1,2,3, \cdots
$$

2) The black hole radius is $R=2 G M / C^{2}$;

3) A black hole has a stable octahedron dark particle formed cold spheres. There is no pressure between the spheres;

4) These octahedron dark particles formed spheres have two poles and fault cones 
which emit black hole energy;

5) Particles circling a black hole wrap around the black hole and form spheres which are ejected out at the poles to form black hole jets.

Additional studies are warranted regarding fault cones, black hole jet, and interactions between two neighboring black holes.

\section{References}

[1] Cao, Z.L. and Cao, H.G. (2013) Unified Field Theory and the Configuration of Particles. International Journal of Physics, 1, 151-161.

[2] Cao, Z.L. and Cao, H.G. (2014) Unified Field Theory and Topology of Nuclei. International Journal of Physics, 2, 15-22. http://dx.doi.org/10.12691/ijp-2-1-4

[3] Cao, Z.L., Cao, H.G. and Qiang, W. (2015) Unified Field Theory and Topology of Atom. American Journal of Modern Physics, 4, 1-7.

[4] Cao, Z.L. and Cao, H.G. (2013) Unified Field Theory. American Journal of Modern Physics, 2, 292-298. http://dx.doi.org/10.11648/j.ajmp.20130206.14

[5] Cao, Z.L. and Cao, H.G. (2013) Unified Field Theory and the Hierarchical Universe. International Journal of Physics, 1, 162-170.

[6] Cao, Z.L. and Cao, H.G. (2013) Non-Scattering Photon Electron Interaction. Physics and Materials Chemistry, 1, 9-12.

[7] Cao, Z.L. and Cao, H.G. (2013) SR Equations without Constant One-Way Speed of Light. International Journal of Physics, 1, 106-109.

[8] Cao, H.G. and Cao, Z.L. (2013) Drifting Clock and Lunar Cycle. International Journal of Physics, 1, 121-127.

[9] Cao, H.G., Cao, Z.L. and Qiang, W. (2015) Nuclear Lattices, Mass and Stability. Open Access Library Journal, 2, e1504. http://dx.doi.org/10.4236/oalib.1101504

[10] Cao, Z.L., Cao, H.G. and Qiang, W. (2015) Theory of Everything. Frontiers of Astronomy Astrophysics and Cosmology, 1, 31-36. http://pubs.sciepub.com/faac/1/1/4

[11] Cao, Z.L. and Cao, H.G. (2013) Unified Field Theory and the Configuration of Particles. International Journal of Physics, 1, 151-161.

[12] Blum, A., Lalli, R. and Renn, J. (2016) The Long Road towards Evidence. Max Planck Society.

[13] (2016) Status of Advanced LIGO Detectors. APS-81st Annual Meeting of the APS Southeastern Section, meetings.aps.org.

[14] (2016) Gravitational Waves Discovered at Long Last. Quanta Magazine. www.quantamagazine.org

[15] McNamara, P. (2016) ESA Congratulations on Gravitational Wave Discovery. ESA. Archived from the Original on 2016-02-11.

[16] Abbott, B. P., (LIGO Scientific Collaboration and Virgo Collaboration) et al. (2016) Observation of Gravitational Waves from a Binary Black Hole Merger. Physical Review Letters, 116, 061102. http://dx.doi.org/10.1103/PhysRevLett.116.061102

[17] Glikman, E., et al. (2015) Major Mergers Host the Most-Luminous Red Quasars at z 2: A Hubble Space Telescope WFC3/IR Study. Astrophysical Journal, 806, 218. http://dx.doi.org/10.1088/0004-637X/806/2/218

[18] http://www.universetoday.com/90714/hubble-telescope-directly-observes-quasar-accretiondisc-surrounding-black-hole/ 
Submit or recommend next manuscript to OALib Journal and we will provide best service for you:

- Publication frequency: Monthly

- 9 subject areas of science, technology and medicine

- Fair and rigorous peer-review system

- Fast publication process

- Article promotion in various social networking sites (LinkedIn, Facebook, Twitter, etc.)

- Maximum dissemination of your research work

Submit Your Paper Online: Click Here to Submit

Or Contact service@oalib.com 